\title{
The Incidence and Predictors of Renal artery Stenosis in Patients Referred for Coronary Angiography
}

\author{
Mohamed Osama Fathy Kayed ${ }^{1}$, Haytham Mahmoud Hassan ${ }^{2}$, Mostafa Elsayed Abd ElGhany ${ }^{1}$, Adham Mohamed Abd \\ Elkader'. \\ ${ }^{1}$ Department of Cardiology and ${ }^{2}$ Department of Community and occupational medicine, Faculty of Medicine. Al-Azhar \\ University, ${ }^{3}$ Department of Cardiology, Matrouh Specialized Cardiothoracic and Interventional Catheterization Center, \\ Egypt. \\ Corresponding author: Adham Mohamed Abd Elkader, Tel: 002-01099452447, dr.adham.abdelkader@gmail.com
}

\begin{abstract}
:
Background: Renal artery stenosis (RAS) and coronary artery disease (CAD) are two manifestations of a same pathogenesis which is atherosclerosis.. RAS is a leading factor of secondary hypertension, ischemic nephropathy and end stage renal disease (ESRD). Aim of work: This study was aimed to detect the incidence and predictors of renal artery stenosis in patients referred for elective coronary angiography. Patients and Methods: In this study we included 100 patients who underwent coronary and renal angiography. The mean age was $56.6+7.9$ years, 60 males (60\%), 40 females (40\%), 60 diabetic (60\%), 58 hypertensive (58\%), 41 smokers (41\%), 62 dyslipidemic (62\%), 27 patients with family history of ischemic heart disease (27\%), and patient with normal coronaries $12(12 \%)$. Our study consisted of 2 groups; 87 (87\%) patients with normal renal arteries (group 1) and $13(13 \%)$ patients with renal artery stenosis (group 2). Results: The incidence of RAS was $13 \%$ and the incidence of significant RAS ( $\geq 50 \%$ stenosis) was $7 \%$. The significant difference between the two groups was in the terms of hypertension ( $\mathrm{p}$-value 0.014 ), female gender (p-value 0.021$)$, multivessel ( $\geq 2$ vessels) coronary artery disease ( $\mathrm{p}$-value 0.046 ), normal coronaries had a significant negative association with RAS (p-value 0.027) and there was a significant relationship between atherosclerotic involvement of Left anterior descending artery (LAD), Diagonal, and right coronary artery (RCA) with RAS (p-value 0.037, 0.041 and 0.042). Conclusion: It could be concluded that predictors of RAS were hypertension, multivessel coronary artery disease ( $\geq 2$ vessels) and female gender.
\end{abstract}

Keywords: Coronary angiography; renal artery stenosis; renal angiography; atherosclerosis.

\section{INTRODUCTION}

Cardiovascular disease is the principal cause of death worldwide. Significantly, it remains the chief cause of preventable death globally. Public health efforts to improve lifestyle and control lifestyle related major cardiovascular risk factors, will definitely contribute to cardiovascular disease prevention ${ }^{[1]}$.

Renal artery stenosis (RAS) generally refers to a disease of the large extra-renal arterial vessels and is mostly caused by atherosclerotic obstructions. Atherosclerotic RAS causes cardiorenal problems. Thus it is a matter of concern for both nephrologists and cardiologists. Yet, the kidney does not present organ-specific signs or symptoms of ischemia, unlike the heart, brain or lower limbs. Successful detection of RAS can thereby be very difficult in clinical practice ${ }^{[2]}$.

Arterial hypertension (HTN), progressive renal failure, flash pulmonary edema, and multivessel coronary disease are clinical manifestations of RAS demanding intervention and treatment and could be resolved by revascularization therapy. In the past, RAS was under recognized, underdiagnosed, and undertreated. With enhanced noninvasive imaging techniques such as magnetic resonance imaging angiography, computed tomography angiography, and high-resolution renal duplex sonography, the diagnosis is now more frequently established ${ }^{[3]}$.

Received: $13 / 10 / 2018$

Accepted: 02/11/2018
Atherosclerosis accounts for about $90 \%$ of cases with RAS and mostly involves the origin and the proximal third of the main renal artery. Actually, ostial RAS can be considered as a combined disease of the aorta and the renal artery, instead of an isolated problem of the renal arteries. ${ }^{[4]}$

Definitely, atherosclerotic RAS is a progressive disease, as more than half of the patients show an increasing degree of stenosis within 5 years after diagnosis ${ }^{[5]}$, and one out of five patients with a critical stenosis undergoes renal atrophy and renal failure throughout this period ${ }^{[3]}$.

\section{Aim of the work:}

This study was aimed to determine the incidence and predictors of renal artery stenosis in patients suspected with coronary artery disease who are referred for coronary angiography. The study was approved by the Ethics Board of Al-Azhar University.

\section{PATIENTS and METHODS}

This prospective study included a total of 100 patients who were subjected to catheterization using the femoral approach. Attending at Matrouh Specialized Cardiothoracic and Interventional Catheterization Center and Al-Azhar (Sayed Galal) University Hospitals. Approval of the ethical committee and a written informed 


\section{The Incidence and Predictors of Renal artery Stenosis in Patients Referred for Coronary Angiography}

consent from all the subjects were obtained. This study was conducted between September 2017 to September 2018.

Before catheterization, all patients underwent routine cardiovascular evaluation to determine medical history, demographics, and atherogenic risk factors.

The study excluded patients with acute coronary syndrome; those with heart failure with reduced ejection fraction (Left ventricular ejection fraction $<40 \%$ ); those with a serum creatinine level $>1.5 \mathrm{mg} /$ $\mathrm{dL}$ and patients who develop any life-threatening complication during the coronary angiography (e.g. pulmonary edema, severe cardiac arrhythmia, unstable angina, etc.).

Catheterization was performed using the femoral approach. All patients underwent left and right coronary angiography using the standard technique. Selective renal arteriography was then performed using a Judkins right catheter inserted consecutively in the ostium of both renal arteries in the left anterior oblique projection with 10- to 20-degree angulation.A dose of 5 to $10 \mathrm{~mL}$ of iso-osmolar iodine containing contrast medium was injected into each renal artery. When the ostia of the renal arteries did not appear clearly, the angulation was modified to obtain better visualization. Angiographic data were individually reviewed, and the degree of stenosis was evaluated by visual estimation.

The result of coronary angiography was classified into: normal CA, one, two, three and four vessel disease. The patient was considered to have multivessel coronary artery disease if having at least $70 \%$ or greater stenosis in at least two major coronary vessels (measuring $\geq 2.5 \mathrm{~mm}$ in diameter), seen in 2 different projections based on visual estimation by two observers. The degree of RAS was recorded and a diameter stenosis $\geq 50 \%$ on the basis of visual estimation was considered significant RAS. R A S was subdivided into mild $(<50 \%)$, moderate $(50 \%-75 \%)$ and severe $(>75 \%)$ RAS.

\section{Statistical analysis}

Recorded data were analyzed using the statistical package for social sciences, version 20.0 (SPSS Inc., Chicago, Illinois, USA). Quantitative data were expressed as mean \pm standard deviation (SD). Qualitative data were expressed as frequency and percentage. Independentsamples t-test of significance was used when comparing between two means. Chi-square $\left(\mathrm{x}^{2}\right)$ test of significance was used in order to compare proportions between two qualitative parameters. The confidence interval was set to $95 \%$ and the margin of error accepted was set to $5 \%$. So, the $\mathrm{P}$-value $<0.05$ was considered significant.

\section{RESULTS}

We enrolled a consecutive subset of 100 (60 male, $60 \%$ - 40 female, $40 \%$ ) patients with a mean age of
$56.65+7.95$ years, 60 diabetic $(60 \%), 58$ hypertensive $(58 \%)$, smokers $41(41 \%)$, dyslipidemic $62(62 \%)$, positive family history of ischemic heart disease 27 (27\%), patient with normal coronaries $12(12 \%)$, patients with one vessel coronary artery disease $15(15 \%)$, patient with two vessel coronary artery disease 33 (33\%), patients with three vessel coronary artery disease 28 $(28 \%)$, patients with four vessel coronary artery disease $12(12 \%) .($ Table 1$)$

Our study group consisted of 2 groups; 87 (87\%) patients with normal renal arteries (group 1) and 13 (13\%) patients with renal artery stenosis (group 2). In our study patients with RAS group subdivided into 4 subgroups as follow $6(6 \%)$ patients with mild $(<50 \%), 6(5 \%)$ patients with moderate $(50 \%-75 \%)$, and $1(1 \%)$ patients with severe $(>75 \%)$ RAS.

Comparison of baseline characteristics among the two study groups revealed that hypertension was significantly higher among group 2 than group 1 $(84.6 \%$ vs $54 \%)$ (p-value 0.014), also female gender was significantly higher in group 2 than group 1 (69.2\% vs $35.6 \%$ ) (p-value 0.021), multivessel coronary artery disease ( $\geq 2$ vessels) was significantly higher among group 2 than group 1 (92.3\% vs $70.1 \%$ ) (p-value 0.046 ), normal coronaries had a significant negative association with group 2 (0\% vs $13.8 \%)$ (p-value 0.027$)$ and there was a significant relationship between atherosclerotic involvement of LAD, Diagonal, and RCA vessels with RAS (p-value 0.037, 0.041 and 0.042).(Table 2)

Table 1. Baseline Characteristics of the Entire study

\begin{tabular}{|lc|}
\hline & $\begin{array}{c}\text { Total study } \\
(\mathbf{n}=100)\end{array}$ \\
\hline Age (year) & $56.65 \pm 7.95$ \\
Male & $60(60 \%)$ \\
Female & $40(40 \%)$ \\
Diabetes mellitus & $60(60 \%)$ \\
Hypertension & $58(58 \%)$ \\
Family history of IHD & $27(27 \%)$ \\
Smoking & $41(41 \%)$ \\
Dyslipidemia & $62(62 \%)$ \\
Serum creatinine (mg/dl) & $1.05 \pm 0.22$ \\
Normal CA / insignicant CAD & $12(12 \%)$ \\
One vessel CAD & $15(15 \%)$ \\
Two vessel CAD & $33(33 \%)$ \\
Three vessel CAD & $28(28 \%)$ \\
$>$ Three vessel CAD & $12(12 \%)$ \\
\hline
\end{tabular}


Table 2. Comparison of the non-RAS and RAS groups according to patient clinical and angiographic characteristics

\begin{tabular}{|c|c|c|c|}
\hline & $\begin{array}{l}\text { No RAS (group 1) } \\
\quad(n=87)\end{array}$ & $\begin{array}{l}\text { RAS (group 2) } \\
\quad(n=13)\end{array}$ & $p$ value \\
\hline Age $\leq 50$ years, $n(\%)$ & $20(23 \%)$ & $1(7.7 \%)$ & 0.206 \\
\hline Age $>50$ years, $n(\%)$ & $67(77 \%)$ & $12(92.3)$ & \\
\hline Male gender, $n(\%)$ & $56(64.4 \%)$ & $4(30.8 \%)$ & \\
\hline Female gender, $n(\%)$ & $31(35.6 \%)$ & $9(69.2 \%)$ & $0.021 *$ \\
\hline Diabetes mellitus, $n(\%)$ & $50(57.5 \%)$ & $10(76.9 \%)$ & 0.182 \\
\hline Hypertension, $n(\%)$ & $47(54 \%)$ & $11(84.6 \%)$ & $0.014 *$ \\
\hline Smoking, $n(\%)$ & $38(43.7 \%)$ & $3(23.1 \%)$ & 0.159 \\
\hline Dyslipidemia, $n(\%)$ & $55(63.2 \%)$ & $7(53.8 \%)$ & 0.516 \\
\hline Family history of IHD & $22(25.3 \%)$ & $5(38.5 \%)$ & 0.318 \\
\hline Serum creatinine (1 mol/L) & $1.05 \pm 0.21$ & $1.09 \pm 0.25$ & 0.494 \\
\hline Normal CA or insignificant CAD & $12(13.8 \%)$ & $0(0 \%)$ & $0.027 *$ \\
\hline One vessel disease, $n(\%)$ & $14(16.1 \%)$ & $1(7.7 \%)$ & 0.952 \\
\hline Multivessel coronary artery disease ( $\geq 2$ vessels), $n(\%)$ & $61(70.1 \%)$ & $12(92.3 \%)$ & $0.046^{*}$ \\
\hline Left main coronary artery disease & $4(4.6 \%)$ & $0(0 \%)$ & 0.430 \\
\hline Left anterior descending coronary artery disease & $53(60.6 \%)$ & $11(84.6 \%)$ & $0.037 *$ \\
\hline Diagonal coronary artery disease & $11(12.6 \%)$ & $4(30.8 \%)$ & $0.041 *$ \\
\hline Left circumflex coronary artery disease & $38(43.7 \%)$ & $4(30.8 \%)$ & 0.379 \\
\hline Obtuse marginal coronary artery disease & $27(31 \%)$ & $6(46.2 \%)$ & 0.280 \\
\hline Right coronary artery disease & $51(58.6 \%)$ & $11(84.6 \%)$ & $0.042 *$ \\
\hline
\end{tabular}

\section{DISCUSSION}

Renal artery stenosis (RAS) is the most common primary disease of the renal arteries. Hypertension and ischemic nephropathy are the most important consequences of renal artery stenosis (RAS) ${ }^{[6]}$. Ischemic nephropathy progresses to end-stage renal disease (ESRD) in $6-17 \%$ of patients ${ }^{[7]}$. RAS is the most common potentially reversible disorder leading to renal replacement therapy [8]. Moreover; the presence of RAS has been independently associated with increased mortality, particularly in patients with coronary artery disease (CAD) and ESRD ${ }^{[9]}$.

RAS is probably under diagnosed despite a substantial prevalence in patients with vascular disease ${ }^{[10]}$. It is commonly found by chance during angiography for other reasons ${ }^{[11]}$.

In the current study the incidence of RAS was $13 \%$.Our findings were concordant with other studies where the reported incidence of RAS varied between $8.2 \%$ and $47 \%$ ${ }^{[12,13]}$ depending on the inclusion criteria. The incidence of significant RAS ( $\geq 50 \%$ stenosis) in our study was $7 \%$ and the reported incidence of significant RAS ( $\geq 50 \%$ stenosis) among patients undergoing coronary angiography ranges from $3.6 \%$ to as high as $28 \%{ }^{[12,13]}$ that wide range of variation of results related to the diff erences in clinical characteristics such as proportion of older patients, prevalence of hypertension, frequency of female gender, ethnicity, the number and the severity of coronary artery lesions. In our study, predictors of RAS were 


\section{The Incidence and Predictors of Renal artery Stenosis in Patients Referred for Coronary Angiography}

hypertension, multivessel coronary artery disease $(\geq 2$ vessels) and female gender.

When coronary angiographic characteristics of the study population were evaluated, there was a significant relationship between multivessel coronary artery disease ( $\geq 2$ vessels) and renal artery stenosis (p-value 0.016) In our study, the incidence of RAS was increased with the increased extent of CAD as follow $0(0 \%)$ patient among RAS group has no diseased coronary vessels, 1 (7.7\%) patients among RAS group have one diseased coronary vessel, 4 (30.7\%) patient among RAS group have two diseased coronary vessel, 5 (38.4\%) patient among RAS group have three diseased coronary vessel, 3 (23.1\%) patients among RAS group have four diseased coronary vessels. Also, there was a significant relationship between atherosclerotic involvement of LAD, Diagonal, and RCA with RAS (p-value 0.037, 0.041 and 0.042).

In agreement with this work, a study by Safiuddin et al. ${ }^{[14]}$ showed that RAS was more common in patients with three vessel CAD compared to those with single or two vessel CAD. They found significant relationships between atherosclerotic involvement of LAD, LCX and RCA and RAS. According to the number of coronaries involved, patients with three vessels CAD showed strong relationship with RAS and those with normal coronaries or insignificant CAD had a potent negative association with RAS. Another study by Santanu et al. ${ }^{[15]}$ showed that there was significant relationship between the number of involved coronary arteries in coronary artery disease and atherosclerotic renal artery stenosis (RAS). Among total 304 CAD patients in this study, no significant RAS were found in single vessel coronary artery disease patients. Only three vessel disease and left main coronary artery disease (TVD/LMCA) disease and two vessel disease coronary artery disease patients had significant renal artery stenosis. Weber et al. ${ }^{[16]}$ concluded that the presence of RAS was particularly high in patients with more than 2 coronary artery lesions. Indian study by Rath et al. [17] showed that highest incidence of RAS was found in patients with three vessel coronary artery disease. A study by Buller et al. ${ }^{[18]}$ showed that significant RAS were found in $19 \%$ of two vessel diease patients, $14 \%$ of three vessel diease patients and 14\% of LMCA disease patients. Harding et. Al. ${ }^{[9]}$ also showed RAS was found mostly in multivessel coronary artery disease.

The results of our study showed that female gender was associated with higher incidence of renal artery stenosis $(\mathrm{p}$ value $=0.021)$. The results are in agreement with many studies $[2,18,19]$ that showed significant relationship between female gender and incidence of RAS. Opposing to our study Ollivier et al. ${ }^{[20]}$ showed that male gender was associated with higher prevalence of renal artery stenosis.

The results of our study showed that there was significant relationship between the incidence of RAS and hypertension ( $\mathrm{p}$ value $=0.014$ ). This was in agreement with majority of the previous studies ${ }^{[16,18,22,23]}$ which found significant relationship between hypertension and increased incidence of RAS. In contrary to our study, Khatami et al. [2] didn't find significant relationship between RAS and HTN.

In the current study there was no significant increase in serum creatinine in patients with RAS which was not in agreement with most of the studies ${ }^{[2,12,13,18,20,22]}$ that showed that there was significant relationship between (increased serum creatinine and decreased glomerular filtration rate) and increased incidence of RAS. That difference between the result of our study and the other studies mostly because all of the studies included patients with serum creatinine levels of $>1.5 \mathrm{mg} / \mathrm{dl}$, but in our study, we excluded patients with serum creatinine of $\geq 1.5$ $\mathrm{mg} / \mathrm{dl}$ for safety purposes.

\section{CONCLUSION}

It could be concluded that the overall incidence of atherosclerotic renal artery stenosis in patients referred for elective coronary angiography was $13 \%$ and the incidence of significant renal artery stenosis was $7 \%$. Predictors of RAS were hypertension, multivessel coronary artery disease ( $\geq 2$ vessels) and female gender. We recommended that renal angiography probably warranted in female, hypertensive patients with multivessel coronary artery disease referred for elective coronary angiography, but not in those with normal coronary arteries. We also recommend that the study should be done on a large scale of patients and on multicenter bases.

\section{REFERENCES}

1. Santulli G (2013): Epidemiology of Cardiovascular Disease in the $21^{\text {st }}$ Century; Updated Numbers and Updated Facts. Journal of Cardiovascular Disease , 1(1):1-2.

2. Khatami MR, Edalati-Fard M, Sadeghian S et al. (2014): Renal artery stenosis in patients with established coronary artery disease: Prevalence and predicting factors. Saudi journal of kidney diseases and transplantation, 25(5): 986-991.

3. Simon JF (2010): Stenting atherosclerotic renal arteries: time to be less aggressive. Cleve Clin J Med., 77(3):178-89.

4. Dubel GJ and Murphy TP (2008): The role of percutaneous revascularization for renal artery stenosis. Vasc Med., 13: 141-56.

5. Rundback JH, Gray RJ, Rozenblit G et al. (2002): Guidelines for the reporting of renal artery revascularization in clinical trials. American Heart Association. Circulation, 106: 1572-1585.

6. Aqel RA, Zoghbi GJ, Baldwin SA et al. (2003): Prevalence of renal Artery stenosis in high-risk 
veterans referred to cardiac catheterization. J Hypertens., 1:1157-62.

7. Cardoso de Carvalho F, Bregagnollo E, Santos Silva V, Brunie A, da Silva Franco RJ, Martin LC, Gavras I, Gavras H (2006): Frequency of coronary artery disease in patients with renal artery stenosis without clinical manifestations of coronary insufficiency. Am J Hypertens., 19: 1125-1128.

8. Weinrauch LA, D'Elia JA (2004): Renal artery stenosis: 'fortuitous diagnosis,' problematic therapy. J Am Col Cardiol., 43: 1614-1616.

9. Harding MB, Smith LR, Himmelstein SI et al. (1992): Renal artery stenosis: Prevalence and associated risk factors in patients undergoing routine cardiac catheterization. J Am SocNephrol., 2:1608-1616.

10. De Silva R, Loh H, Rigby AS, Nikitin NP et al. (2007): Epidemiology, associated factors, and prognostic outcomes of renal artery stenosis in chronic heart failure associated with magnetic resonance angiography. Am J Cardiol., 100:2739

11. Levin A, Linas S, Luft FC et al. (2007): Controversies in renal artery stenosis: A review by the American Society of Nephrology Advisory Group on Hypertension. Am J Nephrol., 2007; 27:212-20.

12. El-Mawardy RH, Magdy AG, Mohsen MM et al. (2008): Prevalence and predictors of renal artery stenosis in hypertensive patients undergoing elective coronary procedures.J ClinHypertens (Greenwich), 10:844-849.

13. Dzielinska Z, Januszewicz A, Demkow M et al. (2007): Cardiovascular risk factors in hypertensive patients with coronary artery disease and coexisting renal artery stenosis. J Hypertens., 25:663-70.

14. Safiuddin M, Ahsan Habib SM, Jamal UM et al. (2012): Angiographic correlation between coronary and renal artery lesions in patients undergoing simultaneous coronary and renal angiography. University Heart Journal, 8: 123-27.
15. Santanu D, Poddar KS, Bandyopadhyay A $e t$ al. (2016): Prevalence and risk profile of atherosclerotic renal artery stenosis in relation to atherosclerotic coronary artery disease.European journal of biomedical and pharmaceutical sciences, 3 (8):178-184.

16. Weber BR, Dieter RS (2014): Renal artery stenosis: epidemiology and treatment. International Journal of Nephrology and Renovascular Disease, 2: 7: 169-181.

17. Rath PC, Agarwala MK, Dhar PK et al. (2002): Renal artery involvement in patients of coronary artery disease undergoing coronary angiography a prospective study. J Assoc Physicians India, 50: 523-6.

18. Buller CE, Nogareda JG, Ramanathan $\mathrm{K}$ et al. (2004): The profile of cardiac patients with renal artery stenosis. J Am CollCardiol., 43: 1606-13.

19. Cohen MG, Pascua JA, Garcia-Ben M et al. (2005): A simple prediction rule for significant renal artery stenosis in patients undergoing cardiac catheterization. Am Heart J., 150: 1204 1211.

20. Ollivier R, Boulmier D, Veillard D et al. (2009): Frequency and predictors of renal artery stenosis in patients with coronary artery disease. CardiovascRevasc Med., 10: 23-29.

21. Alhaddad IA, Blum S, Heller EN et al. (2001): Renal Artery Stenosis in Minority Patients Undergoing Diagnostic Cardiac Catheterization: Prevalence and Risk Factors. J CardiovascPharmacolTher., 6:147-53.

22. Carmelita M, Stefania $R$, Luca $Z$ et al. (2013): Prevalence of renal artery stenosis in patients undergoing cardiac catheterization.Internal and Emergency Medicine, 8 (5): 401-408.

23. Liang F, Hu D, Wu M et al. (2012): The incidence of renal artery stenosis in the patients referred for coronary artery bypass grafting. Indian J Nephrol., 22(1): 13-17. 